The University of San Francisco

USF Scholarship: a digital repository@ Gleeson Library |

Geschke Center

Mathematics

College of Arts and Sciences

3-2009

\title{
Reverse Mathematics, Computability, and Partitions of Trees
}

Jennifer Chubb

University of San Francisco, jennifer.chubb@gmail.com

Jeffry L. Hirst

Timothy H. McNicholl

Follow this and additional works at: http://repository.usfca.edu/math

Part of the Mathematics Commons

\section{Recommended Citation}

Chubb, Jennifer; Hirst, Jeffry L.; and McNicholl, Timothy H., "Reverse Mathematics, Computability, and Partitions of Trees" (2009). Mathematics. Paper 4.

http://repository.usfca.edu/math/4

This Article is brought to you for free and open access by the College of Arts and Sciences at USF Scholarship: a digital repository @ Gleeson Library | Geschke Center. It has been accepted for inclusion in Mathematics by an authorized administrator of USF Scholarship: a digital repository @ Gleeson Library| Geschke Center. For more information, please contact repository@usfca.edu. 


\title{
REVERSE MATHEMATICS, COMPUTABILITY, AND PARTITIONS OF TREES
}

\author{
JENNIFER CHUBB, JEFFRY L. HIRST, AND TIMOTHY H. MCNICHOLL
}

\author{
Abstract. We examine the reverse mathematics and computability theory of a form of Ramsey's theorem \\ in which the linear $n$-tuples of a binary tree are colored.
}

Let $2^{<\mathbb{N}}$ denote the full binary tree of height $\omega$. We identify nodes of the tree with finite sequences of zeros and ones, and refer to any subset of the nodes as a subtree. For positive integers $n$, let $\left[2^{<\mathbb{N}}\right]^{n}$ denote the set of all linearly ordered $n$-tuples of nodes in $2^{<\mathbb{N}}$. We say that a subtree $S$ of $2^{<\mathbb{N}}$ is isomorphic to $2^{<\mathbb{N}}$ if every node of $S$ has exactly two immediate successors. More formally, $S \subseteq 2^{<\mathbb{N}}$ is isomorphic to $2^{<\mathbb{N}}$ if there is a bijective function $f: 2^{<\mathbb{N}} \rightarrow S$ such that for all $\sigma, \tau \in 2^{<\mathbb{N}}$, we have $\sigma \subseteq \tau$ if and only if $f(\sigma) \subseteq f(\tau)$. This weak form of isomorphism does not preserve minima. Using this terminology, we can formulate the following version of Ramsey's theorem for trees.

$\mathrm{TT}_{k}^{n}$ : Suppose that $\left[2^{<\mathbb{N}}\right]^{n}$ is colored with $k$ colors. Then there is a subtree $S$ isomorphic to $2^{<\mathbb{N}}$ such that $[S]^{n}$ is monochromatic.

Although we have not found this principle stated verbatim in the literature, $\mathrm{TT}_{k}^{1}$ is an immediate consequence of Theorem 1.3 of [8] and also of Theorem 2.1 of [2]. In his dissertation [7], McNicholl used $\mathrm{TT}_{k}^{1}$ to find the combinatorial conditions that are necessary and sufficient to carry out a kind of priority construction. Iterated applications of Theorem 2.3 of [2] could be used to prove $\mathrm{TT}_{k}^{n}$ for all finite $n$ and $k$. All these cited results use a stronger notion of isomorphism than is used in our formulation.

The goal of this paper is to examine the reverse mathematics and computability theory of this form of Ramsey's theorem. Section 1 gives the reverse mathematical analysis of $\mathrm{TT}_{k}^{n}$ and consequently includes proofs of $\mathrm{TT}_{k}^{n}$. Section 2 proves upper and lower bounds on the complexity of the monochromatic sets, paralleling the similar bounds proved by Jockusch in [5] for Ramsey's theorem on the integers. We conclude the paper by noting extensions of these results to other infinite trees and listing some questions.

§1. Reverse mathematics. In this section, we analyze $\mathrm{TT}_{k}^{n}$ using the hierarchy of subsystems of second order arithmetic detailed in Simpson's book [10]. We need

Received July 24, 2007.

2000 Mathematics Subject Classification. 03B30, 03D80, 05C55, 03F35, 03 E02.

Key words and phrases. Ramsey, tree, arithmetical, comprehension, bounds. 
only three systems. The base system RCA $A_{0}$ includes induction restricted to $\Sigma_{1}^{0}$ sets and a form of set comprehension for computable sets. Our proof of $\mathrm{TT}_{k}^{1}$ uses $\mathrm{RCA}_{0}$ with induction for $\Sigma_{2}^{0}$ formulas appended. Proofs for higher exponents require the use of $A C A_{0}$, which adds comprehension for arithmetically definable sets to $R C A_{0}$. We begin by carrying out a proof of $\mathrm{TT}_{k}^{1}$ in a weak system.

Lemma 1.1. $\left(\mathrm{RCA}_{0}\right)$ Let $f: 2^{<\mathbb{N}} \rightarrow$ red, blue $\}$ be a two coloring of the nodes of the full binary tree. For any node $\sigma$ of the tree either (1) above $\sigma$ there is a subtree isomorphic to $2^{<\mathbb{N}}$ in which every nonempty node is colored red, or (2) $\sigma$ can be extended to a node $\tau$ such that every node properly extending $\tau$ is colored blue.

Proof. Suppose $f$ and $\sigma$ are as hypothesized. Enumerate the pairs of nodes of $2^{<\mathbb{N}}$. Construct the red tree as follows. Let $p_{\langle\rangle}=\sigma$. Given $p_{\alpha}$, let $p_{\alpha \frown 0}$ and $p_{\alpha \prec 1}$ be the first pair of incomparable red nodes extending $p_{\alpha}$. If this process never fails, then $\left\{p_{\alpha} \mid \alpha \in 2^{<\mathbb{N}}\right\}$ is the desired red tree. If the process fails, then there is a least node $\beta \supset \sigma$ such that all red extensions of $\beta$ are comparable. If no extension of $\beta$ is red, let $\tau=\beta$. Every proper extension of $\tau$ is blue. If there is a node $\alpha \supset \beta$ such that $\alpha$ is red, pick the least such node and write $\alpha=\beta^{\urcorner} \gamma^{\urcorner} \varepsilon$, where $\varepsilon \in\{0,1\}$. Then let $\tau=\beta^{\frown} \gamma^{\frown}(1-\varepsilon)$ and note that every proper extension of $\tau$ is blue.

In the following, we will refer to the red tree in the proof of Lemma 1.1 as the standard red subtree (of $2^{<\mathbb{N}}$ for $\sigma$ using $f$ ). We will refer to the blue subtree as the full subtree (of $2^{<\mathbb{N}}$ for $\sigma$ ). The proof of the next result uses induction on $\Sigma_{2}^{0}$ formulas. Consequently, the statement of the theorem refers to $R C A_{0}+\Sigma_{2}^{0}$-IND.

THEOREM 1.2. ( $\left(\mathrm{RCA}_{0}+\Sigma_{2}^{0}\right.$-IND) For all $k, \mathrm{TT}_{k}^{1}$. That is, for any finite coloring of $2^{<\mathbb{N}}$, there is a monochromatic subtree isomorphic to $2^{<\mathbb{N}}$.

Proof. Suppose $f: 2^{<\mathbb{N}} \rightarrow k$ is a finite coloring of the nodes of $2^{<\mathbb{N}}$. Consider the set $C=\{j<k \mid \exists \sigma \forall \tau(\tau \supseteq \sigma \rightarrow j \leq f(\tau))\}$. By bounded $\Sigma_{2}^{0}$ comprehension, which is provable from $\Sigma_{2}^{0}$-IND in $\mathrm{RCA}_{0}$ (see [10], page 72), the set $C$ exists. Now $0 \in C$, so $C$ is nonempty and finite. Find the largest element of $C$ and call it $j$. Since $j \in C$, there must be a witness $\sigma \in 2^{<\mathbb{N}}$ such that $\forall \tau(\tau \supseteq \sigma \rightarrow j \leq f(\tau))$. Consider the two coloring on extensions of $\sigma$ defined by $g(\tau)=$ red if $f(\tau)=j$ and $g(\tau)=$ blue otherwise. The existence of a full blue subtree for $g$ contradicts the choice of $j$ as maximal. Consequently, Lemma 1.1 shows there is a standard red subtree for $g$ above $\sigma$, which is an isomorphic copy of $2^{<\mathbb{N}}$ on which $f$ constantly takes the value $j$.

It is easy to deduce the infinite pigeonhole principle from $\mathrm{TT}_{k}^{1}$; simply color nodes according to their level. The infinite pigeonhole principle is equivalent to the bounding principle $B \Pi_{1}^{0}$ (see [3] or [1]) which is strictly weaker than $\Sigma_{2}^{0}$-IND. Thus, the exact strength of $\mathrm{TT}_{k}^{1}$ is at least $\mathrm{B} \Pi_{1}^{0}$ and at most $\Sigma_{2}^{0}$-IND. To carry out a proof of $\mathrm{TT}_{k}^{n}$, we need to prove $\mathrm{TT}_{k}^{2}$ as a base case.

THeOREm 1.3. $\left(\mathrm{ACA}_{0}\right)$ For all $k, \mathrm{TT}_{k}^{2}$. That is, for any finite coloring of pairs of comparable nodes of $2^{<\mathbb{N}}$, there is a monochromatic subtree isomorphic to $2^{<\mathbb{N}}$.

Proof For two COLORS ONLY. Suppose $f:\left[2^{<\mathbb{N}}\right]^{2} \rightarrow\{$ red, blue $\}$ is a two coloring of pairs of comparable nodes of the full binary tree. Given any $\sigma \in 2^{<\mathbb{N}}$, we define an induced map on single nodes $f_{\sigma}:\left\{\tau \in 2^{<\mathbb{N}} \mid \tau \supset \sigma\right\} \rightarrow 2$ by setting $f_{\sigma}(\tau)=f(\sigma, \tau)$. 
Define $p_{\sigma}, T_{\sigma}$, and $c_{\sigma}$ as follows. Set $p_{\langle\rangle}=\langle\rangle$and $T_{\langle\rangle}=2^{<\mathbb{N}}$. Suppose $p_{\sigma}$ and $T_{\sigma}$ have been defined. If there is a full blue subtree of $T_{\sigma}$ for $p_{\sigma}$ using $f_{p_{\sigma}}$, then make the following assignments:

- $c_{\sigma}=$ blue.

- Let $p_{\sigma \frown 0}$ and $p_{\sigma \frown 1}$ be the first two nonempty nodes of the full blue subtree of $T_{\sigma}$ for $p_{\sigma}$ using $f_{p_{\sigma}}$.

- For $\varepsilon \in\{0,1\}$, set $T_{\sigma \frown \varepsilon}=\left\{\tau \in T_{\sigma} \mid \tau \supseteq p_{\sigma^{\wedge} \varepsilon}\right\}$.

If there is no full blue subtree of $T_{\sigma}$ for $p_{\sigma}$ using $f_{p_{\sigma}}$, then make the following assignments:

- $c_{\sigma}=$ red.

- Let $p_{\sigma \frown 0}$ and $p_{\sigma \frown 1}$ be the first two nonempty nodes of the standard red tree of $T_{\sigma}$ for $p_{\sigma}$ using $f_{p_{\sigma}}$.

- For $\varepsilon \in\{0,1\}, T_{\sigma \wedge \varepsilon}$ consists of those nodes of the standard red tree of $T_{\sigma}$ for $p_{\sigma}$ using $f_{p_{\sigma}}$ which extend $p_{\sigma \frown \varepsilon}$.

Let $S=\left\{p_{\sigma} \mid \sigma \in 2^{<\mathbb{N}}\right\}$. Since $p_{\sigma}$ is arithmetically definable from the values of $p_{\tau}$ and $c_{\tau}$ for $\tau \subset \sigma, \mathrm{ACA}_{0}$ proves the existence of $S$. By the construction, whenever $\sigma \subset \tau \in 2^{<\mathbb{N}}$, we have $f\left(p_{\sigma}, p_{\tau}\right)=c_{\sigma}$. The map $p_{\sigma} \mapsto c_{\sigma}$ is a 2 coloring of $S$ whose existence is provable in $\mathrm{ACA}_{0}$. Since $A C A_{0}$ implies $\Sigma_{2}^{0}$-IND, an application of Theorem 1.2 yields a color $c$ and a subtree $T$ of $S$ such that $p_{\sigma} \in T$ implies $c_{\sigma}=c$. Consequently, if $p_{\sigma} \subset p_{\tau}$ are elements of $T$, then $f\left(p_{\sigma}, p_{\tau}\right)=c$, completing the proof.

Proof OF THeOREM 1.3 FOR $k$ COLORS. Suppose $f:\left[2^{<\mathbb{N}}\right]^{2} \rightarrow k$ is a finite coloring of pairs of comparable nodes of $2^{<\mathbb{N}}$. Given any $\sigma \in 2^{<\mathbb{N}}$, we define an induced map on single nodes $f_{\sigma}:\left\{\tau \in 2^{<\mathbb{N}} \mid \tau \supset \sigma\right\} \rightarrow k$ by setting $f_{\sigma}(\tau)=f(\sigma, \tau)$.

Define $p_{\sigma}, T_{\sigma}$, and $c_{\sigma}$ as follows. Set $p_{\langle\rangle}=\langle\rangle$and $T_{\langle\rangle}=2^{<\mathbb{N}}$. Given $p_{\sigma}$ and $T_{\sigma}$, define $c_{\sigma}$ as follows. Let $j$ be the least integer such that there is no $p \supset p_{\sigma}$ in $T_{\sigma}$ such that $\forall \tau \in T_{\sigma}\left(\tau \supset p \rightarrow j<f_{p_{\sigma}}(\tau)\right)$. Since $j$ is the least such integer, there is a $p \supset p_{\sigma}$ in $T_{\sigma}$ such that $\forall \tau \in T_{\sigma}\left(\tau \supset p \rightarrow j \leq f_{p_{\sigma}}(\tau)\right)$. Fix this $p$, and note that by the definition of $j$, there is no $q \supset p$ in $T_{\sigma}$ such that $\forall \tau \in T_{\sigma}\left(\tau \supset q \rightarrow j<f_{p_{\sigma}}(\tau)\right)$. If we treat the color $j$ as red and the colors greater than $j$ as blue, by Lemma 1.1, the standard $j$-colored subtree of $T_{\sigma}$ for $p$ using $f_{p_{\sigma}}$ exists and is isomorphic to $2^{<\mathbb{N}}$. Call this tree $T$. Let $c_{\sigma}=j$. Let $p_{\sigma \frown 0}$ and $p_{\sigma \frown 1}$ be the two level one elements of $T$. For $\varepsilon \in\{0,1\}$, let $T_{\sigma \frown \varepsilon}$ be the subtree of T with root $p_{\sigma \frown \varepsilon}$.

Let $S=\left\{p_{\sigma} \mid \sigma \in 2^{<\mathbb{N}}\right\}$. Since $p_{\sigma}$ is arithmetically definable from the values of $p_{\tau}$ and $c_{\tau}$ for $\tau \subset \sigma, \mathrm{ACA}_{0}$ proves the existence of $S$. By the construction, whenever $\sigma \subset \tau \in 2^{<\mathbb{N}}$, we have $f\left(p_{\sigma}, p_{\tau}\right)=c_{\sigma}$. The map $p_{\sigma} \mapsto c_{\sigma}$ is a finite coloring of $S$ whose existence is provable in $\mathrm{ACA}_{0}$. Since $A C A_{0}$ implies $\Sigma_{2}^{0}$-IND, an application of Theorem 1.2 yields a color $c$ and a subtree $T$ of $S$ such that $p_{\sigma} \in T$ implies $c_{\sigma}=c$. Consequently, if $p_{\sigma} \subset p_{\tau}$ are elements of $T$, then $f\left(p_{\sigma}, p_{\tau}\right)=c$, completing the proof.

We complete the proof of $\mathrm{TT}_{k}^{n}$ in $\mathrm{ACA}_{0}$ using the following inductive step. We abbreviate $\forall k\left(\mathrm{TT}_{k}^{n}\right)$ by $\mathrm{TT}^{n}$.

TheOREM 1.4. $\left(\mathrm{ACA}_{0}\right)$ For all $n \geq 1, \mathrm{TT}^{n}$ implies $\mathrm{TT}^{n+1}$.

Proof. We will generalize the proof of Theorem 1.3 to handle higher exponents by constructing a subtree $S$ such that the color of any $n+1$-tuple is determined by 
its first $n$ elements, and applying $\mathrm{TT}^{n}$ to $S$ to obtain the desired monochromatic tree. Suppose $f:\left[2^{<\mathbb{N}}\right]^{n+1} \rightarrow k$ is a finite coloring of the $n+1$-tuples of comparable nodes of $2^{<\mathbb{N}}$. If $P=\left\{p_{\tau} \mid \tau \subseteq \sigma\right\}$ is a sequence of comparable nodes terminating in $p_{\sigma}$, we define an induced coloring of single nodes $\tau \supset p_{\sigma}$ by setting

$$
f_{p_{\sigma}}(\tau)=\prod_{\vec{m} \in[P]^{n}} \operatorname{pr}(\vec{m})^{f(\vec{m}, \tau)}
$$

where if $r$ is the integer code for the sequence $\vec{m}$, then $\operatorname{pr}(\vec{m})$ is the $r^{\text {th }}$ prime. Note that $f_{p_{\sigma}}$ uses no more than $k^{|P|^{n}}$ colors.

Define $p_{\sigma}, T_{\sigma}$, and $c_{\sigma}$ as follows. Set $p_{\langle\rangle}=\langle\rangle$and $T_{\langle\rangle}=2^{<\mathbb{N}}$. Given $p_{\sigma}$ and $T_{\sigma}$, let $c_{\sigma}$ be the greatest integer in the range of $f_{p_{\sigma}}$ such that there is a $p \supset p_{\sigma}$ in $T_{\sigma}$ such that

$$
\forall \tau \in T_{\sigma}\left(\tau \supset p \rightarrow c_{\sigma} \leq f_{p_{\sigma}}(\tau)\right) .
$$

Fix the least such $p$, and note that the standard $c_{\sigma}$-colored subtree of $T_{\sigma}$ for $p$ using $f_{p_{\sigma}}$ exists and is isomorphic to $2^{<\mathbb{N}}$. Call this tree $T$. Let $p_{\sigma \sim 0}$ and $p_{\sigma \wedge 1}$ be the two level one elements of $T$ and let $T_{\sigma \frown \varepsilon}$ be the subtree of $T$ with root $p_{\sigma \frown \varepsilon}$ for $\varepsilon \in\{0,1\}$.

Since $p_{\sigma}$ is arithmetically definable from the values of $p_{\tau}$ and $c_{\tau}$ for $\tau \subset \sigma, \mathrm{ACA}_{0}$ proves the existence of the tree $S=\left\{p_{\sigma} \mid \sigma \in 2^{<\mathbb{N}}\right\}$. By the construction of $S$, given any increasing sequence of elements of $S$ of the form

$$
p_{1} \subset p_{2} \subset \cdots \subset p_{n} \subset p_{n+1} \subset p_{n+2},
$$

we have $f_{p_{n}}\left(p_{n+1}\right)=f_{p_{n}}\left(p_{n+2}\right)$, and so $f\left(p_{1}, \ldots, p_{n}, p_{n+1}\right)=f\left(p_{1}, \ldots, p_{n}, p_{n+2}\right)$. Consequently, the function $g:[S]^{n} \rightarrow k$ defined for $p_{\sigma_{1}} \subset \cdots \subset p_{\sigma_{n}}$ by

$$
g\left(p_{\sigma_{1}}, \ldots, p_{\sigma_{n}}\right)=f\left(p_{\sigma_{1}}, \ldots, p_{\sigma_{n}}, p_{\sigma_{n} 0}\right)
$$

indicates the color of any $n+1$-tuple extending $\left(p_{\sigma_{1}}, \ldots, p_{\sigma_{n}}\right)$. By $\mathrm{TT}^{n}$ there is a subtree of $S$ which is isomorphic to $2^{<\mathbb{N}}$, monochromatic for $g$, and so also monochromatic for $f$.

The use of $A C A_{0}$ in the preceding results is necessary, as shown by the reversal included in the following theorem.

THEOREM 1.5. For $n \geq 3$ and $k \geq 2, \mathrm{RCA}_{0}$ proves that the following are equivalent:

(1) $\mathrm{ACA}_{0}$.

(2) $\mathrm{TT}^{n}$.

(3) $\mathrm{TT}_{k}^{n}$.

Proof. Theorems 1.3 and 1.4 show that (1) implies (2). Since (3) is a special case of (2), it remains only to show that (3) implies (1). Note that (3) implies Ramsey's theorem for $n$-tuples and two colors by the following argument. If $g:[\mathbb{N}]^{n} \rightarrow 2$, then we may define $f:\left[2^{<\mathbb{N}}\right]^{n} \rightarrow 2$ by setting $f\left(\sigma_{1}, \ldots, \sigma_{n}\right)=g\left(\operatorname{lh}\left(\sigma_{1}\right), \ldots, \operatorname{lh}\left(\sigma_{n}\right)\right)$. From any monochromatic subtree for $f$, we can construct an infinite monochromatic set for $g$. Whenever $n \geq 3$, Ramsey's theorem for $n$-tuples and two colors implies $\mathrm{ACA}_{0}$ (see Lemma III.7.5 of [10]), completing the proof.

We have shown that $\mathrm{TT}_{2}^{3}$ implies $\mathrm{ACA}_{0}$, but the exact strength of $\mathrm{TT}^{2}$ and $\mathrm{TT}_{2}^{2}$ remain open. Using the level coloring argument of Theorem 1.5, it easy to show that $\mathrm{TT}^{2}$ implies Ramsey's theorem for pairs, but whether or not the converse is provable in $\mathrm{RCA}_{0}$ remains open. See Section 3 for more comments on this. 
$\S 2$. Computability theory. This section turns to computability theory, presenting bounds on the complexity of the monochromatic sets paralleling those of [5]. Since every coloring of $[\mathbb{N}]^{n}$ induces a coloring of $\left[2^{<\mathbb{N}}\right]^{n}$ via the level coloring technique in the proof of Theorem 1.5, Theorem 5.1 of [5] yields the following theorem.

THEOREM 2.1. If $n \geq 2$ then there is a computable 2-coloring of $\left[2^{<\mathbb{N}}\right]^{n}$ with no $\Sigma_{n}^{0}$ monochromatic subtree.

A $\Pi_{2}^{0}$ bound. The proofs of the corresponding upper bound results are significantly more involved. Consider the following result, which is analogous to Theorem 4.2 of [5].

THEOREM 2.2. Every computable finite coloring of pairs of comparable nodes of $2^{<\mathbb{N}}$ has a $\Pi_{2}^{0}$ monochromatic subtree that is isomorphic to $2^{<\mathbb{N}}$.

PROOF. We will carry out the proof for two colors, and then indicate how to extend the result to an arbitrary finite number of colors. Suppose $f:\left[2^{<\mathbb{N}}\right]^{2} \rightarrow\{$ red, blue $\}$ is a computable two coloring of the pairs of comparable nodes of $2^{<\mathbb{N}}$. Any computable monochromatic subtree would be $\Pi_{2}^{0}$ definable, so for the remainder of the proof we may assume no computable monochromatic subtree exists.

Emulating the proof of Jockusch's Theorem 4.2 in [5], we will show that the complement of the desired monochromatic subtree is computably enumerable in $0^{\prime}$ and then apply the strong hierarchy theorem [9]. Initially, we will need to enumerate the complement of an analog of the tree $S$ in the proof of Theorem 1.3. This enumeration, also computable from $0^{\prime}$, will be built using moving markers.

Intuitively, the markers used in this proof eventually settle on nodes corresponding to the $p_{\sigma}$ nodes of $S$ in Theorem 1.3. By making initial guesses at the associated colors and allowing for later revisions, we can execute the construction using only a $0^{\prime}$ oracle. In this respect, this proof closely follows Jockusch's proof. However, arranging for the monochromatic tree to be isomorphic to $2^{<\mathbb{N}}$ complicates the selection of the nodes, especially when the color blue is assigned to a node.

As in the proof of Theorem 1.3, for each $\alpha \in 2^{<\mathbb{N}}$ we will have a marker $p_{\alpha}$. We write $p_{\alpha}^{s}$ to indicate the location of $p_{\alpha}$ at stage $s$. Similarly, we will use the colors $c_{\alpha}^{s} \in\{$ red, blue $\}$. At each stage, if $\alpha \subset \beta$ and $p_{\alpha}^{s}$ and $p_{\beta}^{s}$ are in use, we require $p_{\alpha}^{s} \subset p_{\beta}^{s}$ and $f\left(p_{\alpha}^{s}, p_{\beta}^{s}\right)=c_{\alpha}^{s}$. As before, we will frequently write $f_{p_{\alpha}}\left(p_{\beta}\right)$ for $f\left(p_{\alpha}, p_{\beta}\right)$.

At each stage $s$, we will also have a finite set $M^{s}$ consisting of all $\alpha \in 2^{<\mathbb{N}}$ such that $p_{\alpha}$ is in use, and a finite set $E^{s}$ which is a correct initial segment of the complement of the analog of $S$. ( $M$ is for map and $E$ is for ejected.) In selecting locations for newly introduced markers, we will be careful to avoid elements of $E^{s}$.

For each $\alpha \in 2^{<\mathbb{N}}$ and stage $s$, we will also have a tree $T_{\alpha}^{s}$ of possible extensions of $p_{\alpha}^{s}$. Just as we intend for $p_{\alpha}^{s}$ to converge to a node in the analog of $S$, we intend for $T_{\alpha}^{s}$ to converge to a tree isomorphic to $2^{<\mathbb{N}}$. However, $T_{\alpha}^{s}$ may be a finite tree at some stages, due to erroneous selections. Regardless of the size of $T_{\alpha}^{s}$, it is completely described by $E^{s}$ together with a finite sequence of pairs called a descriptor. Descriptors are defined inductively as follows. The sequence of no pairs, \langle\rangle , is a descriptor for $2^{<\mathbb{N}}$. Writing $d\left(T_{\alpha}^{s}\right)$ for the descriptor of $T_{\alpha}^{s}$, if $p \in T_{\alpha}^{s}$ then $d\left(T_{\alpha}^{s}\right)^{\wedge}(p$, red $)$ is the descriptor for the tree obtained by following the algorithm for constructing the standard red subtree of $T_{\alpha}^{s}$ for $p$ using $f_{p}$, avoiding all nodes in $E^{s}$. In executing the algorithm to find the standard red subtree, we will assume that 
all elements at level $k$ are determined before any elements at level $k+1$. Similarly, $d\left(T_{\alpha}^{s}\right)^{\frown}(p$, full $)$ is the descriptor for the tree of all elements of $T_{\alpha}^{s}$ extending the least extension of $p$ which lies above all elements of $E^{s}$. Call this least extension the root. Because of the way we will construct $E^{s}$, the root of the tree with descriptor $d\left(T_{\alpha}^{s}\right) \frown(p$, full) is always a proper extension of $p$. Because of the way descriptors are defined, for any $s$ and $\alpha, T_{\alpha}^{s}$ is either isomorphic to $2^{<\mathbb{N}}$ or finite. Since descriptors are always finite, they can be encoded by an integer and tagged onto markers.

In the following construction, the behavior of each marker is very limited. Initially, we place $p_{\alpha}$ and guess that red is the appropriate color for $c_{\alpha}$. As long as no difficulties arise in locating extensions of $p_{\alpha}$ in standard red subtrees, $p_{\alpha}$ and $c_{\alpha}$ remain unchanged. If the search for extensions fails, then (some) $p_{\alpha}$ must have a full blue subtree for $f_{p_{\alpha}}$. In this case, we change $c_{\alpha}$ to blue and attempt to move $p_{\alpha}$ to a successor of its current location. This move is a necessary complication, allowing us to decode a monochromatic subtree from the analog of $S$. If $c_{\alpha}$ is blue at stage $s$, then $p_{\alpha}$ will not be moved unless the descriptor $d\left(T_{\alpha}^{s}\right)$ is shortened or for some $\beta \subset \alpha, p_{\beta}$ is modified.

Stage 0: Let $p_{\langle\rangle}^{0}=\langle\rangle, c_{\langle\rangle}^{0}=$ red, $d\left(T_{\langle\rangle}^{0}\right)=(\langle\rangle$, red $), E^{0}=\emptyset$, and $M^{0}=\{\langle\rangle\}$. Thus, we have assigned the empty node the color red, will search for successors of this marker in the standard red subtree of $2^{<\mathbb{N}}$ for \langle\rangle using $f_{\langle\rangle}$, have determined no elements in the complement of the analog of $S$, and have placed exactly one marker, corresponding to the location of \langle\rangle in $2^{<\mathbb{N}}$. All other markers are unassigned.

Stage $s+1$ : We will use two cases to describe the action at this stage.

Case 1: For each leaf $\beta \in M^{s}$, we can locate incomparable proper extensions $p_{\beta, 0}$ and $p_{\beta, 1}$ of $p_{\beta}^{s}$ in $T_{\beta}^{s}$. Note that we can determine whether or not this case holds on the basis of finitely many queries to $0^{\prime}$. When this case holds, do the following:

- For each leaf $\beta \in M^{s}$ and each $\varepsilon \in\{0,1\}$ :

- Set $p_{\beta\urcorner \varepsilon}^{s+1}=p_{\beta, \varepsilon}$ and $c_{\beta\urcorner \varepsilon}^{s+1}=$ red;

- add all elements of $M^{s}$ and $\beta^{\wedge} \varepsilon$ to $M^{s+1}$;

- Let the descriptor for $T_{\beta>\varepsilon}^{s+1}$ be $d\left(T_{\beta}^{s}\right) \frown\left(p_{\beta, \varepsilon}\right.$, red $)$.

- For all other $\alpha$, set $p_{\alpha}^{s+1}=p_{\alpha}^{s}, c_{\alpha}^{s+1}=c_{\alpha}^{s}$, and $d\left(T_{\alpha}^{s+1}\right)=d\left(T_{\alpha}^{s}\right)$.

- Let $L=\max \left\{\operatorname{lh}\left(p_{\alpha}^{s+1}\right) \mid \alpha \in M^{s+1}\right\}$, and set

$$
E^{s+1}=E^{s} \cup\left\{\tau \in 2^{<\mathbb{N}} \mid \operatorname{lh}(\tau)<L \wedge \forall \alpha \in M^{s+1}\left(\tau \neq p_{\alpha}^{s+1}\right)\right\} .
$$

Case 2: Case 1 fails, so there is a leaf $\beta \in M^{s}$ with no incomparable proper extensions of $p_{\beta}^{s}$ in $T_{\beta}^{s}$. Using $0^{\prime}$ we can fix such a leaf $\beta$.

Intuitively, whenever this situation arises, we need to create a blue marker. For example, if $c_{\beta}^{s}$ is red and we can find no such extensions, then we should change $c_{\beta}^{s}$ to blue. Though not as obvious, blue markers with no extensions arise from erroneous red nodes in descriptors. To complicate matters, simply changing the color of a marker creates problems with extracting the final monochromatic tree from our analog of $S$. Consequently, in this case we will move some marker and color it blue.

We will search each tree $B$ in a list for a pair of nodes $p_{0} \subset p_{1}$ such that $\forall \tau \in B\left(p_{1} \subset \tau \rightarrow f_{p_{0}}(\tau)=\right.$ blue $)$. The trees fall into two categories. If $\alpha \subseteq \beta$ and $c_{\alpha}^{s}=$ red, then the descriptor $d\left(T_{\alpha}^{s}\right)$ is of the form $d^{\frown}\left(p_{\alpha}^{s}\right.$, red). For each (possibly 
empty) sequence $p_{\alpha, 0} \subset p_{\alpha, 1} \subset \cdots \subset p_{\alpha, k}$ of nodes in the tree with descriptor $d^{\frown}\left(p_{\alpha}^{s}\right.$, full $)$, add the tree with descriptor

$$
d^{\frown}\left(p_{\alpha}^{s}, \text { full }\right)^{\frown}\left(p_{\alpha, 0}, \text { red }\right)^{\frown} \ldots \frown\left(p_{\alpha, k}, \text { red }\right)
$$

to the list. If $\alpha \subseteq \beta$ and $c_{\alpha}^{s}=$ blue, then the descriptor $d\left(T_{\alpha}^{s}\right)$ may be of the form $d^{\frown}\left(p_{\alpha, 0}\right.$, red $) \frown \ldots \frown\left(p_{\alpha, k}\right.$, red $) \frown\left(p_{\alpha, k+1}\right.$, full $) \frown\left(p_{\alpha, k+2}\right.$, full $)$ where $k \geq 0$. If so, then for each $j \leq k$ add the tree with the descriptor

$$
d^{\frown}\left(p_{\alpha, 0}, \text { red }\right)^{\frown} \ldots \frown\left(p_{\alpha, j-1}, \text { red }\right)^{\frown}\left(p_{\alpha, j}, \text { full }\right)
$$

to the list. Search all trees in the list until $p_{0}$ and $p_{1}$ as described at the beginning of this paragraph are found. (We allow $p_{0}$ to be the root of a tree; in particular, if the descriptor of $B$ terminates in $\left(p_{\alpha, j}\right.$, full), we may let $p_{0}$ be the root of $B$, which is the least extension of $p_{\alpha, j}$ lying above all elements of $E^{s}$.) A proof that this search always terminates is given in Claim 1 below. Remember the descriptor of the tree for which the search succeeded, including the value of $\alpha$.

Suppose we have found $p_{0}, p_{1}$, and $\alpha \subseteq \beta$ as specified in the preceding paragraph. Do the following:

- Let $M^{s+1}=\left\{\gamma \in M^{s} \mid \gamma \not \supset \alpha\right\}$.

- For $\gamma \in M^{s+1}-\{\alpha\}$, let $p_{\gamma}^{s+1}=p_{\gamma}^{s}, c_{\gamma}^{s+1}=c_{\gamma}^{s}$, and $d\left(T_{\gamma}^{s+1}\right)=d\left(T_{\gamma}^{s}\right)$.

- Let $E^{s+1}=E^{s}$.

Denote the descriptor of the tree for which the search succeeded by $d_{0}$ and do the following:

- Set $c_{\alpha}^{s+1}=$ blue and $p_{\alpha}^{s+1}=p_{0}$.

- Let $d\left(T_{\alpha}^{s+1}\right)=d_{0}^{\curlyvee}\left(p_{0}\right.$, full $) \frown\left(p_{1}\right.$, full $)$.

This completes the construction. The next four claims show that the construction yields the desired enumeration of the complement of the analog of $S$.

Claim 1: The search described in Case 2 of Stage $s+1$ always terminates.

Proof of Claim 1: Suppose there is a leaf $\beta \in M^{s}$ with no proper extensions of $p_{\beta}^{s}$ in $T_{\beta}^{s}$. The absence of extensions indicates that $T_{\beta}^{s}$ is not isomorphic to $2^{<\mathbb{N}}$, so $T_{\beta}^{s}$ must be finite. Each initial segment of the descriptor $d\left(T_{\beta}^{s}\right)$ is a descriptor for some tree. Since the empty sequence is the descriptor for $2^{<\mathbb{N}}$, there must be a first pair $(p, c)$ such that the initial segment of $d\left(T_{\beta}^{s}\right)$ terminating in $(p, c)$ describes a finite tree.

If $d$ is the descriptor for a tree isomorphic to $2^{<\mathbb{N}}$ containing $p$, then $d^{\frown}(p$, full) is also isomorphic to $2^{<\mathbb{N}}$. Thus the pair $(p, c)$ in the preceding paragraph must be of the form $\left(p\right.$, red). The node $p$ must either be a $p_{\alpha}$ for some $\alpha \subseteq \beta$, or a node on a path leading to some $p_{\alpha}$ for which $c_{\alpha}^{s}=$ blue. We will consider these situations in order.

First suppose $(p, c)$ is of the form $\left(p_{\alpha}\right.$, red) for some $\alpha \subseteq \beta$ where $c_{\alpha}^{s}=$ red. Then $d\left(T_{\beta}^{s}\right)$ is of the form $d^{\frown}\left(p_{\alpha}^{s} \text {, red }\right)^{\wedge} \hat{d}$. (Note that the following holds when $\hat{d}=\emptyset$.) The tree with descriptor $d^{\frown}\left(p_{\alpha}^{s}\right.$, full $)$ is isomorphic to $2^{<\mathbb{N}}$. Suppose by way of contradiction that the search fails. That is, given any (possibly empty) sequence $p_{\alpha, 0} \subset p_{\alpha, 1} \subset \cdots \subset p_{\alpha, k}$ of nodes in the tree with descriptor $d^{\frown}\left(p_{\alpha}^{s}\right.$, full), if we let $B$ be the tree with descriptor

$$
d^{\frown}\left(p_{\alpha}^{s}, \text { full }\right)^{\frown}\left(p_{\alpha, 0}, \text { red }\right)^{\frown} \ldots \frown\left(p_{\alpha, k}, \text { red }\right),
$$


then there is no pair $p_{0} \subset p_{1}$ in $B$ such that $\forall \tau \in B\left(p_{1} \subset \tau \rightarrow f_{p_{0}}(\tau)=\right.$ blue $)$. Consequently, for any such $B, p_{0}$, and $p_{1}$, there is a $\tau \supset p_{1}$ in $B$ such that $f_{p_{0}}(\tau)=$ red. We can use this feature to construct a computable monochromatic red tree as follows.

Let $B$ denote the tree with descriptor $d^{\frown}\left(p_{\alpha}^{s}\right.$, full $)$. Let $q_{\langle\rangle}$denote the root of this tree; that is, $q_{\langle\rangle}$is the least extension of $p_{\alpha}^{s}$ lying above all elements of $E^{s}$. By the preceding paragraph, there is no $p_{1} \supset q_{\langle\rangle}$such that $\forall \tau \in B\left(p_{1} \subset \tau \rightarrow\right.$ $f_{q_{\langle\rangle}}(\tau)=$ blue $)$. Let $B_{\langle\rangle}$be the tree with descriptor $d^{\frown}\left(q_{\langle\rangle}\right.$, red $)$. By Lemma 1.1, $B_{\langle\rangle}$is isomorphic to $2^{<\mathbb{N}}$. Suppose $q_{\alpha}$ and $B_{\alpha}$ are defined and $B_{\alpha}$ is isomorphic to $2^{<\mathbb{N}}$. Let $q_{\alpha \frown 0}$ and $q_{\alpha-1}$ be the first pair of incomparable elements of $B_{\alpha}$. For each $\varepsilon \in\{0,1\}$, treating $q_{\alpha^{-} \varepsilon}$ as $p_{0}$, by the preceding paragraph, the tree with descriptor

$$
d^{\frown}\left(q_{\langle\rangle}, \text {red }\right)^{\frown} \ldots\left(q_{\alpha}, \text { red }\right)^{\frown}\left(q_{\alpha \frown \varepsilon}, \text { red }\right)
$$

(which will be $B_{\alpha^{-} \varepsilon}$ ) is isomorphic to $2^{<\mathbb{N}}$. Note that if $\alpha \subset \beta$, then $q_{\beta} \in B_{\alpha}$, so $f_{q_{\alpha}}\left(q_{\beta}\right)=$ red. Thus $\left\{q_{\alpha} \mid \alpha \in 2^{<\mathbb{N}}\right\}$ is a computable monochromatic tree for $f$.

The existence of a computable monochromatic tree for $f$ contradicts the first paragraph of the proof of this theorem. Consequently, when $c_{\alpha}^{s}$ is red, the search must terminate, completing the proof for this situation.

Now suppose $(p, c)$ is of the form $\left(p_{\alpha, j}\right.$, red) for some $\alpha \subseteq \beta$ where $c_{\alpha}^{s}=$ blue. Then $d\left(T_{\beta}^{s}\right)$ is of the form

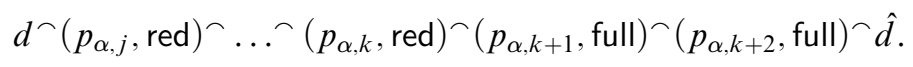

(Note that the following holds if $\hat{d}=\emptyset$ and also if $j=k$.) Since $\left(p_{\alpha, j}\right.$, red) was the first pair yielding a finite tree, the tree with descriptor $d^{\frown}\left(p_{\alpha, j}\right.$, full $)$ is isomorphic to $2^{<\mathbb{N}}$. As in the preceding paragraphs, if we cannot find $p_{0}$ and $p_{1}$ satisfying the search, then we can construct a computable monochromatic tree, yielding a contradiction. This completes the proof of the claim that the search always succeeds.

Claim 2: For every $\alpha \in 2^{<\mathbb{N}}$, the following limits exist: $\lim _{s} p_{\alpha}^{s}=p_{\alpha}, \lim _{s} c_{\alpha}^{s}=$ $c_{\alpha}$, and $\lim _{s} d\left(T_{\alpha}^{s}\right)=d_{\alpha}$.

Proof of Claim 2: Consider the possible behaviors for $p_{\langle\rangle}^{s}$. If $p_{\langle\rangle\rangle}^{s}$ is never moved, then $p_{\langle\rangle}=\langle\rangle, c_{\langle\rangle}=$red, and $T_{\langle\rangle}$is the tree with descriptor ( \langle\rangle , red). At some stage $s, p_{\langle\rangle}^{s}$ may be moved, in which case $c_{\langle\rangle}^{s}=$ blue and $T_{\langle\rangle}^{s}$ has a new descriptor of some length $n$. At any successive stage $t, c_{\langle\rangle}^{t}=$ blue and if $p_{\langle\zeta\rangle}^{t}$ moves, then the descriptor of $T_{\langle\rangle}^{t}$ is shortened. Consequently, the process must eventually converge to a limiting $p_{\alpha}$ and $d_{\alpha}$.

If $p_{\alpha}, c_{\alpha}$ and $d_{\alpha}$ have achieved their limits at stage $s$, then the only allowable changes in $p_{\alpha \frown \varepsilon}^{t}, c_{\alpha\urcorner \varepsilon}^{t}$, and $d\left(T_{\alpha \succ \varepsilon}^{t}\right)$, for $\varepsilon \in\{0,1\}$ and $t>s$ are exactly those in the preceding paragraph. Thus, all the markers must achieve their limits.

Furthermore, each time Case 2 of Stage $s+1$ is executed, either $M^{s}$ is decreased in size, or for some $\alpha \in M^{s}$, either $c_{\alpha}^{s}$ is changed from red to blue or the descriptor of $T_{\alpha}^{s}$ is shortened. Since $M^{s}$ and all descriptors are finite, Case 1 of Stage $s+1$ must occur infinitely often. Consequently, once $p_{\alpha}$ achieves its limit, $p_{\alpha \frown \varepsilon}^{s}$ must eventually be introduced, and will also achieve its limit. Thus, for every $\alpha \in 2^{<\mathbb{N}}$, $p_{\alpha}$ and $c_{\alpha}$ are assigned, and $T_{\alpha}$ is a nonempty tree. 
Claim 3: For every $\alpha \in 2^{<\mathbb{N}}$, if $\alpha \subset \beta$ then $p_{\alpha} \subset p_{\beta}, d_{\alpha} \subset d_{\beta}$, and $f_{p_{\alpha}}\left(p_{\beta}\right)=c_{\alpha}$. Proof of Claim 3: Detailed examination of the construction shows that for each $s$, if $\alpha \subset \beta \in M^{s}$, then $p_{\alpha}^{s} \subset p_{\beta}^{s}$, $p_{\beta}^{s} \in T_{\alpha}^{s}$, and $d\left(T_{\beta}^{s}\right)$ extends $d\left(T_{\alpha}^{s}\right)$. Consequently, $f_{p_{\alpha}^{s}}\left(p_{\beta}^{s}\right)=c_{\alpha}^{s}$ and $T_{\beta}^{s} \subset T_{\alpha}^{s}$. Since these relationships are preserved at each stage, they must hold in the limit.

Claim 4: $\overline{\left\{p_{\alpha} \mid \alpha \in 2^{<\mathbb{N}}\right\}}=\bigcup_{s} E^{s}$.

Proof of Claim 4: As shown in Claims 2 and 3, for each $\alpha \in 2^{<\mathbb{N}}, p_{\alpha}$ exists, and if $\beta \supset \alpha$, then $p_{\beta} \supset p_{\alpha}$. Thus, the length of $p_{\alpha}^{s}$ can be forced to exceed any fixed bound in $\mathbb{N}$ by picking suitably large values of $s$ and $\alpha$. By virtue of the definition of $E^{s+1}$ in Case 1 of Stage $s+1$ (which occurs infinitely often), $\bigcup_{s} E_{s} \supseteq \overline{\left\{p_{\alpha} \mid \alpha \in 2^{<\mathbb{N}}\right\}}$. Since each $T_{\alpha}^{s}$ is defined so as to avoid elements of $E^{s}$, no $p_{\alpha}$ can be an element of $\bigcup_{s} E_{s}$.

Summarizing the proof to this point, we have a subtree $\left\{p_{\alpha} \mid \alpha \in 2^{<\mathbb{N}}\right\}$ which is isomorphic to $2^{<\mathbb{N}}$, and satisfies $f_{p_{\alpha}}\left(p_{\beta}\right)=c_{\alpha}$ whenever $\alpha \subset \beta$. Furthermore, the complement of this set is the union of finite sets each of which can be computed with the aid of $0^{\prime}$. Consequently, the complement is computably enumerable in $0^{\prime}$. Thus, we have found an analog of $S$ whose complement is c.e. in $0^{\prime}$. It remains to extract a monochromatic subtree and describe an enumeration for its complement.

First, suppose there is an $\alpha$ such that for all $\beta \supseteq \alpha, c_{\beta}=$ red. Then the subtree $T=\left\{p_{\beta} \mid \beta \supseteq \alpha\right\}$ is the desired monochromatic red tree. To enumerate the complement of $T$, repeat the construction, adding $p_{\gamma}^{s}$ to $E^{s}$ whenever $p_{\gamma}^{s} \not \supset p_{\alpha}$. Since the complement of $T$ is computably enumerable in $0^{\prime}$, by the strong hierarchy theorem $T$ is $\Pi_{2}^{0}$ definable.

Finally, suppose that for every $\alpha$ there is a $\beta \supset \alpha$ such that $c_{\beta}=$ blue. We repeat the construction, adding new markers $\left\{t_{\alpha} \mid \alpha \in 2^{<\mathbb{N}}\right\}$, new finite subsets of the complement of the monochromatic tree $\left\{F^{s} \mid s \in \mathbb{N}\right\}$, and new maps $\left\{N^{s} \mid s \in \mathbb{N}\right\}$ where $N^{s}$ contains those $\alpha$ for which $t_{\alpha}$ is attached at stage $s$.

Run the construction until the first $c_{\alpha}^{s}$ is set to blue. Let $t_{\langle\rangle}^{s}=p_{\alpha}^{s}, N^{s}=\{\langle\rangle\}$, and $F^{s}=\left\{p_{\beta}^{s} \mid \beta \in M^{s} \wedge \beta \neq \alpha\right\}$. Note that if $p_{\beta}^{s} \in F^{s}$, then $c_{\beta}^{s}=$ red.

At stage $s+1$, execute the process for constructing $S$, and then consider three cases.

Case 1: For each leaf $\beta \in N^{s}$, given that $t_{\beta}^{s}=p_{\gamma}^{s}$, suppose we can locate extensions $p_{\delta_{0}}^{s} \supset p_{\gamma \sim 0}^{s}$ and $p_{\delta_{1}}^{s} \supset p_{\gamma \wedge 1}^{s}$ such that $c_{\delta_{0}}^{s}=$ blue and $c_{\delta_{1}}^{s}=$ blue. In this case, do the following:

- For each leaf $\beta \in N^{s}$ and each $\varepsilon \in\{0,1\}$,

$$
\begin{aligned}
& \text { - set } t_{\beta^{\prime} \varepsilon}^{s+1}=p_{\delta_{\varepsilon}}^{s} \text {, and } \\
& \text { - add all elements of } N^{s} \text { and } \beta^{\wedge} \varepsilon \text { to } N^{s+1} \text {. }
\end{aligned}
$$

- For all other $\alpha \in N^{s}$, set $t_{\alpha}^{s+1}=t_{\alpha}^{s}$.

- Define $F^{s+1}$ by the equation

$$
F^{s+1}=F^{s} \cup\left\{\tau \in 2^{<\mathbb{N}} \mid \exists \alpha \in M^{s+1}\left(\tau=p_{\alpha}^{s+1}\right) \wedge \forall \alpha \in N^{s+1}\left(\tau \neq t_{\alpha}^{s+1}\right)\right\} .
$$

Case 2: For some $\beta \in N^{s}$, a predecessor of $t_{\beta}^{s}$ is moved. Let $p_{\delta}^{s}$ be this predecessor node. Because of the way nodes are added in Case 1, there is a unique least $\alpha \subseteq \beta$ such that $t_{\alpha}^{s} \supseteq p_{\delta}^{s}$. Find this $\alpha$, and do the following:

- Let $N^{s+1}=\left\{\gamma \in N^{s} \mid \gamma \not \supset \alpha\right\}$. 
- For $\gamma \in N^{s+1}-\{\alpha\}$, let $t_{\gamma}^{s+1}=t_{\gamma}^{s}$.

- Let $F^{s+1}=F^{s}$.

- Let $t_{\alpha}^{s+1}=p_{\delta}^{s+1}$.

This last step is possible because $p_{\delta}^{s}$ was moved to a previously unassigned location, guaranteeing that $p_{\delta}^{s} \notin F^{s}$. Furthermore, since $p_{\delta}^{s}$ moved, $c_{\delta}^{s+1}=$ blue.

Case 3: If neither Case 1 nor Case 2 holds, let $N^{s+1}=N^{s}, F^{s+1}=F^{s}$, and $t_{\alpha}^{s+1}=t_{\alpha}^{s}$ for all $\alpha \in N^{s+1}$.

It is not difficult to show that for each $\alpha$, the limit $t_{\alpha}=\lim _{s} t_{\alpha}^{s}$ exists, and that it marks some $p_{\gamma}$ such that $c_{\gamma}=$ blue. Also, $\overline{\left\{t_{\alpha} \mid \alpha \in 2^{<\mathbb{N}}\right\}}=\bigcup_{s} E_{s} \cup \bigcup_{s} F_{s}$, so $\left\{t_{\alpha} \mid \alpha \in 2^{<\mathbb{N}}\right\}$ is the complement of a set which is computably enumerable in $0^{\prime}$. Thus, in this situation, $\left\{t_{\alpha} \mid \alpha \in 2^{<\mathbb{N}}\right\}$ is a blue monochromatic tree which is $\Pi_{2}^{0}$ definable.

We have completed the proof for two colors. To extend the result to an arbitrary finite number of colors, we modify the construction, assigning colors 0 through $k$ in order. Initially $c_{\alpha}^{s}$ is assigned 0 . In Case 2 of Stage $s+1$, if $c_{\alpha}^{s}$ is assigned $j<k$ then we search for $p_{0} \subset p_{1}$ such that $\forall \tau \in B\left(p_{1} \subset \tau \rightarrow j<f_{p_{0}}(\tau)\right)$ and set $c_{\alpha}^{s+1}=j+1$. The color $k$ behaves like blue in the original construction.

The claims are proved as before, yielding an analog of $S$ with each $c_{\alpha}$ in $\{0, \ldots, k\}$. Pick the least $j$ such that there is an $\alpha$ such that for all $\beta \supseteq \alpha, c_{\beta} \leq j$. If $j=0$, then $T=\left\{p_{\beta} \mid \beta \supseteq \alpha\right\}$ is the desired monochromatic subtree. Otherwise, rerun the construction using new markers to extract a $j$-colored subtree. The $\Pi_{2}^{0}$ bounds follow as before.

The $\Pi_{n}^{0}$ bound. In the proof of Theorem 2.7, the preceding theorem acts as a base case for deducing the bounds for colorings of $n+1$-tuples. In the argument, our goal is to produce a subtree with a controlled level of complexity such that the coloring of any $n+1$-tuple depends only on the first $n$ elements. The color blocks defined below aid in controlling the complexity of the desired tree. In all of the following, let $f: 2^{<\mathbb{N}} \rightarrow k$. Also, for each $\alpha \in 2^{<\mathbb{N}}$, we let $T_{\alpha}$ denote the full subtree of $2^{<\mathbb{N}}$ extending $\alpha$, that is $T_{\alpha}=\left\{\tau \in 2^{<\mathbb{N}} \mid \alpha \subseteq \tau\right\}$.

Definition 1. A color block for $f$ is a set of $k+1$ chains with the following properties:

1. Each chain consists of $k$ nodes, exactly one of each color.

2. Any two nodes chosen from distinct chains are incomparable.

Definition 2. For $c<k$, we say $f$ has a full $c$-avoiding tree if there is some node $\tau$ such that for all $\sigma \supset \tau, f(\sigma) \neq c$.

LEMMA 2.3. Either there is a $c$ such that $f$ has a full $c$-avoiding tree or there is a color block for $f$.

Proof. We search $2^{<\mathbb{N}}$ for a color block for $f$. If the search fails, it is because we have discovered a full $c$-avoiding tree for some $c$.

Begin by selecting $k+1$ pairwise incomparable nodes in $2^{<\mathbb{N}}$, the least $k+1$ such nodes will do. For each node $\sigma$ in this collection, do the following:

Let $\sigma_{0}=\sigma$. For $0 \leq i \leq k-2$, given $\sigma_{i}$, let $\sigma_{i+1}$ be the least node extending $\sigma_{i}$ with $f\left(\sigma_{i+1}\right) \neq f\left(\sigma_{j}\right)$ for $j \leq i$, if such a node exists. 
(Note that establishing the existence of such a $\sigma_{i+1}$ requires a query to $0^{\prime}$ when $f$ is computable.)

If this search fails for some $i$, it is because all nodes $\tau$ extending $\sigma_{i}$ have $f(\tau)=$ $f\left(\sigma_{j}\right)$ for some $j \leq i$, thus $T_{\sigma_{i}}$ is $c$-avoiding for any $c \notin\left\{f\left(\sigma_{j}\right) \mid j \leq i\right\}$.

If the search does not fail, we have successfully completed the construction of $k+1$ non-intersecting chains, each consisting of $k$ distinctly colored nodes. Further, when $f$ is computable, this construction may be carried out with only finitely many queries to $0^{\prime}$.

Definition 3. If $f$ is a $k$-coloring and $S$ is a subset of the colors, an $S$ color block for $f$ is a collection of $|S|+1$ chains, each of which is composed of exactly one node from each color in $S$, satisfying the incomparability requirement in the definition of a color block.

Given a coloring of $\left[2^{<\mathbb{N}}\right]^{n+1}$ and an $\alpha \in 2^{<\mathbb{N}}$, we can color nodes of $T_{\alpha}$ by fixing $n$-tuples at or below $\alpha$ and assigning colors to each node above $\alpha$. We present some definitions and lemmas about collections of colorings which could be induced in this fashion. Intuitively, the $\left\langle f_{\alpha}\right\rangle$-forest defined below consists of finite approximations to the tree used for reducing $n+1$-tuples to $n$-tuples.

For what follows, assume that for each $\alpha \in 2^{<\mathbb{N}}$, the function $f_{\alpha}: T_{\alpha} \rightarrow k_{\alpha}$ is a finite coloring of $T_{\alpha}$. For a node $\alpha$ of length $n$, denote the initial segments of $\alpha$ by \langle\rangle$=\alpha_{0} \subset \alpha_{1} \subset \alpha_{2} \subset \cdots \subset \alpha_{n}=\alpha$. Using this notation, define $k_{\alpha}^{*}=\left\{\left(j_{0}, \ldots, j_{n}\right) \mid \forall i j_{i}<k_{\alpha_{i}}\right\}$, and the functions $f_{\alpha}^{*}: T_{\alpha} \rightarrow k_{\alpha}^{*}$ by $f_{\alpha}^{*}(\tau)=$ $\left(f_{\alpha_{0}}(\tau), f_{\alpha_{1}}(\tau), \ldots, f_{\alpha_{n}}(\tau)\right)$.

Definition 4. An $\left\langle f_{\alpha}\right\rangle$-forest is defined in terms of a sequence of levels $\left\langle L_{i}\right\rangle_{i \in \mathbb{N}}$. The levels are defined as follows: Let $L_{0}=\{\langle\rangle\}$. Note that $f_{\langle\rangle}^{*}=f_{\langle\rangle}$and that the range of $f_{\langle\rangle}$is $\left\{0,1, \ldots, k_{\langle\rangle}-1\right\}$. Attach the $\operatorname{tag}\left(\left\{0,1, \ldots, k_{\langle\rangle}-1\right\},\langle\rangle\right)$ to \langle\rangle .

Suppose that $L_{n}$ is defined. If some $\sigma \in L_{n}$ has a tag, then do the following:

1. If the tag on $\sigma$ is $(S, \tau)$ and $|S|>1$, then check for an $S$ color block for $f_{\sigma}^{*}$ above $\tau$ using the algorithm from the proof of Lemma 2.3.

(a) If such a color block is located, add all the nodes in the color block to $L_{n+1}$. Whenever $\mu$ is the supremum of a chain in the color block, define $S_{\mu}=\left\{\left(v_{0}, v_{1}, \ldots, v_{|\sigma|}, \ldots v_{|\mu|}\right) \in k_{\mu}^{*} \mid\left(v_{0}, v_{1}, \ldots, v_{|\sigma|}\right) \in S\right\}$ and attach the $\operatorname{tag}\left(S_{\mu}, \mu\right)$ to $\mu$. Remove the tag from $\sigma$.

(b) If no such color block is found, then for some $c \in S$ and some $\beta$ above $\tau$ there is a $c$-avoiding tree for $f_{\sigma}^{*}$ above $\beta$. Change the tag on $\sigma$ to $(S-\{c\}, \beta)$.

2. If the tag on $\sigma$ is $(S, \tau)$ and $|S|=1$, then the tree above $\tau$ is monochromatic for $f_{\sigma}^{*}$. Add $\tau^{\frown} 0$ and $\tau^{\frown} 1$ to $L_{n+1}$. For each $\varepsilon \in\{0,1\}$, define $S_{\tau^{\wedge} \varepsilon}=\left\{\left(v_{0}, \ldots, v_{|\sigma|}, \ldots, v_{\left|\tau^{\wedge} \varepsilon\right|}\right) \in k_{\tau^{\prime} \varepsilon}^{*} \mid\left(v_{0}, \ldots, v_{\sigma}\right) \in S\right\}$ and attach the $\operatorname{tag}\left(S_{\tau^{\wedge} \varepsilon}, \tau^{\wedge} \varepsilon\right)$ to $\tau^{\wedge} \varepsilon$. Remove the tag from $\sigma$.

If no element of $L_{n}$ has a tag, then the calculation of $L_{n+1}$ is complete, and $L_{n+1}$ is defined. Note that this process always terminates, and that for each $n, L_{n}$ is finite.

The $\left\langle f_{\alpha}\right\rangle$-forest consists of all finite binary subtrees $T$ such that:

(1) the $k^{\text {th }}$ level of $T$ is empty or contains exactly $2^{k}$ elements from $L_{k}$, and

(2) if $\sigma, \tau_{1}$, and $\tau_{2}$ are nodes of $T$ and $\tau_{1}$ and $\tau_{2}$ both extend $\sigma$, then $f_{\sigma}^{*}\left(\tau_{1}\right)=$ $f_{\sigma}^{*}\left(\tau_{2}\right)$. 
Observe that by requiring (2), we ensure that when $T$ is a tree in an $\left\langle f_{\alpha}\right\rangle$-forest and $\tau$ is in $T$, then $f_{\tau}$ is monochromatic on the nodes of $T$ above $\tau$. Lemmas 2.4, 2.5, and 2.6 complete the construction of the tree needed for the proof of Theorem 2.7. Let $[T]$ denote some canonical integer code for a finite tree $T$.

Lemma 2.4. Suppose that for each $\alpha \in 2^{<\mathbb{N}}, f_{\alpha}: T_{\alpha} \rightarrow k_{\alpha}$ is a finite coloring of $T_{\alpha}$. If $\left\langle f_{\alpha}\right\rangle_{\alpha \in 2^{<N}}$ is a computable collection of computable finite colorings, then the $\left\langle f_{\alpha}\right\rangle$-forest is computable from $0^{\prime}$. Furthermore, there is a function $g$ such that $g \leq 0^{\prime}$ and for all $n$, if $T$ is a height $n$ element of the $\left\langle f_{\alpha}\right\rangle$-forest, then $[T] \leq g(n)$.

Proof. Let $T$ be a finite tree of height $n$, and $\mathscr{F}$ the $\left\langle f_{\alpha}\right\rangle$-forest. To determine whether $T \in \mathscr{F}$, we first (computably) check that it satisfies the monochromaticity requirement in (2) in the definition above and is isomorphic to $2 \leq n$. Then check for each $k \leq n$ that

$$
(\forall \sigma \in T)\left[\sigma \text { has } k \text { predecessors in } T \Longrightarrow \sigma \in L_{k}\right] .
$$

Thus membership in $\mathscr{F}$ reduces to finitely many questions about membership in the sets $L_{k}$ for $k \leq n$. By the proof of Lemma 2.3, the construction of the (finite) set $L_{k}$ can be carried out with the assistance of $0^{\prime}$.

Since each set $L_{k}$ is finite, there are only finitely many trees of a given height $n$ belonging to $\mathscr{F}$. With the aid of $0^{\prime}$, we may find these and set $g(n)$ to exceed the largest of their canonical indices.

LEMMA 2.5. If $\left\langle f_{\alpha}\right\rangle$ is as in Lemma 2.4, then there is a subtree $T$ such that the following hold:

1. $T$ is isomorphic to $2^{<\mathbb{N}}$.

2. For each $\sigma \in T, f_{\sigma}$ is constant on $\{\tau \in T \mid \tau \supset \sigma\}$.

3. $T^{\prime} \leq 0^{\prime \prime}$.

Proof. Let $\mathscr{F}$ denote the $\left\langle f_{\alpha}\right\rangle$-forest and order its elements by inclusion. By Lemma $2.4, \mathscr{F}$ has the structure of a finitely branching tree bounded by a function $g$, and both $\mathscr{F}$ and $g$ can be computed from $0^{\prime}$.

Once we show below that $\mathscr{F}$ is infinite, we can apply the relativized Low Basis Theorem [6] to obtain from $\mathscr{F}$ a path $P$ such that $P^{\prime} \leq 0^{\prime \prime}$. The desired tree $T$ is the union of the elements in this path. A node of length $n$ on the path is a finite tree isomorphic to $2^{\leq n}$ and they form a nested (increasing) sequence, so their union $T$ is indeed isomorphic to $2^{<\mathbb{N}}$. Since $T \leq P$ and $P^{\prime} \leq 0^{\prime \prime}$, we have $T^{\prime} \leq 0^{\prime \prime}$.

Note that the definition of an $\left\langle f_{\alpha}\right\rangle$-forest ensures that for all $\sigma \in T, f_{\sigma}^{*}$ is constant on $\{\tau \in T \mid \tau \supset \sigma\}$ and so $f_{\sigma}$ is also constant on this set.

To see that $\mathscr{F}$ is infinite suppose by way of contradiction that there is an upper bound on the height of elements of $\mathscr{F}$. In this case, we may find a sequence of colorings $\left\langle h_{\alpha}\right\rangle$ such that the maximum height of a tree in the $\left\langle h_{\alpha}\right\rangle$-forest is minimal among all choices of colorings. Let $H$ be a tree from the $\left\langle h_{\alpha}\right\rangle$-forest that has this maximal height, which we will denote by $n$. It follows from Lemma 2.3 and the definition of $\left\langle f_{\alpha}\right\rangle$-forest that $n \geq 1$. Let $L_{1}$ be the first level of the $\left\langle h_{\alpha}\right\rangle$-forest. We consider two cases.

First suppose $L_{1}$ consists of nodes taken from a monochromatic subtree for $h_{\langle\rangle}$; denote these by $\tau_{0}$ and $\tau_{1}$. For each nonempty $\alpha \in 2^{<\mathbb{N}}$, define $h_{\alpha}^{\tau_{0}}$ by $h_{\alpha}^{\tau_{0}}(\beta)=$ $h_{\tau_{0} \alpha}\left(\tau_{0}^{\frown} \beta\right)$ and also define $h_{\langle\rangle}^{\tau_{0}}(\beta)=h_{\tau_{0}}^{*}\left(\tau_{0}^{\curlyvee} \beta\right)$. Define $h_{\alpha}^{\tau_{1}}$ similarly, and note that for $i \in\{0,1\}$ the trees of the $\left\langle h_{\alpha}^{\tau_{i}}\right\rangle$-forest are the extensions of $\tau_{i}$ in the $\left\langle h_{\alpha}\right\rangle$-forest. 
By our choice of $n$, the $\left\langle h_{\alpha}^{\tau_{0}}\right\rangle$-forest and the $\left\langle h_{\alpha}^{\tau_{1}}\right\rangle$-forest each contain a tree of height $n$; call them $T_{\tau_{0}}$ and $T_{\tau_{1}}$. Then $\{\langle\rangle\} \cup T_{\tau_{0}} \cup T_{\tau_{1}}$ is a tree in the $\left\langle h_{\alpha}\right\rangle$-forest of height $n+1$, contradicting the choice of $n$.

Now suppose $L_{1}$ consists of nodes in a color block for $h_{\langle\rangle}$and let $\mu_{0}, \ldots, \mu_{j}$ be the maximal elements of the $j+1$ chains in $L_{1}$. Note here that the cardinality of the range of $h_{\langle\rangle}$on nodes in and above the chains is $j$. As in the previous paragraph, construct the induced sequences of colorings for each $\mu_{i}$, and a monochromatic tree $M_{\mu_{i}}$ of height $n$ for each $\mu_{i}$. Two of these, say $M_{\mu_{i}}$ and $M_{\mu_{j}}$, must agree in the first component of their coloring. Pick $\sigma_{i}$ in the chain below $\mu_{i}$ so that $\left\{\sigma_{i}\right\} \cup\left(M_{\mu_{i}}-\left\{\mu_{i}\right\}\right)$ is monochromatic for $h_{\langle\rangle}$. Choose $\sigma_{j}$ for $M_{\mu_{j}}$ similarly, and note that $\left\{\langle\rangle, \sigma_{i}, \sigma_{j}\right\} \cup\left(M_{\mu_{i}}-\left\{\mu_{i}\right\}\right) \cup\left(M_{\mu_{j}}-\left\{\mu_{j}\right\}\right)$ is a tree of height at least $n+1$ in the $\left\langle h_{\alpha}\right\rangle$-forest, contradicting the choice of $n$ and completing the proof that $\mathscr{F}$ is infinite.

Lemma 2.6. Suppose $n>1$ and $f:\left[2^{<\mathbb{N}}\right]^{n+1} \rightarrow k$ is computable. There is a tree $T$ which is isomorphic to $2^{<\mathbb{N}}$ such that the following hold:

1. $T^{\prime} \leq 0^{\prime \prime}$.

2. If $\sigma_{1}, \ldots, \sigma_{n}$ is a sequence of $n$ comparable elements of $T$ and $\tau_{1}$ and $\tau_{2}$ are extensions of $\sigma_{n}$, then $f\left(\sigma_{1}, \ldots, \sigma_{n}, \tau_{1}\right)=f\left(\sigma_{1}, \ldots, \sigma_{n}, \tau_{2}\right)$.

Proof. Define a computable family of colorings $\left\langle f_{\alpha}\right\rangle_{\alpha \in 2^{<N}}$ as follows. If $\operatorname{lh}(\alpha)<$ $n$, let $f_{\alpha}(\tau)=0$ for all $\tau$. If $\operatorname{lh}(\alpha) \geq n$, let $\vec{\sigma}_{1}, \ldots, \vec{\sigma}_{m}$ be an enumeration of the $n$-tuples of nodes at or below $\alpha$. For $\tau \supset \alpha$, let $f_{\alpha}(\tau)=\prod_{j \leq m} \operatorname{pr}\left(\vec{\sigma}_{j}\right)^{f\left(\vec{\sigma}_{j}, \tau\right)}$, where $\operatorname{pr}(\vec{\sigma})$ denotes the $r$ th prime for some canonical code $r$ for $\vec{\sigma}$.

Apply Lemma 2.5 to $\left\langle f_{\alpha}\right\rangle$ to obtain a tree $T$ isomorphic to $2^{<\mathbb{N}}$ with $T^{\prime} \leq 0^{\prime \prime}$. Let $\vec{\gamma}$ denote an increasing $n$-tuple $\gamma_{1}, \ldots, \gamma_{n}$ of comparable elements of $T$. By Lemma 2.5, $f_{\gamma_{n}}$ is constant on $\left\{\tau \in T \mid \tau \supset \gamma_{n}\right\}$. Let $\tau_{1}, \tau_{2} \in T$ extend $\gamma_{n}$. Then $f_{\gamma_{n}}\left(\tau_{1}\right)=f_{\gamma_{n}}\left(\tau_{2}\right)$. From the definition of $f_{\gamma_{n}}$, the prime power factors corresponding to the $n$-tuple $\vec{\gamma}$ must agree, yielding:

$$
\operatorname{pr}(\vec{\gamma})^{f\left(\vec{\gamma}, \tau_{1}\right)}=\operatorname{pr}(\vec{\gamma})^{f\left(\vec{\gamma}, \tau_{2}\right)} .
$$

The exponents in the preceding equation must match, so $f\left(\vec{\gamma}, \tau_{1}\right)=f\left(\vec{\gamma}, \tau_{2}\right)$ as desired.

Finally, we have assembled all the machinery to prove the analog of Theorem 5.5 in [5].

THEOREM 2.7. If $f:\left[2^{<\mathbb{N}}\right]^{n} \rightarrow k$ is computable, then there is a $\Pi_{n}^{0}$ monochromatic subtree isomorphic to $2^{<\mathbb{N}}$.

Proof. Essentially quoting the proof of Theorem 5.5 of [5], we use induction on $n$. The case $n=1$ follows from Theorem 1.2 and $n=2$ follows from Theorem 2.2. Suppose the theorem holds for some $n \geq 2$, we will prove it for $n+1$. Let $f:\left[2^{<\mathbb{N}}\right]^{n+1} \rightarrow k$ be computable. Find $T$ as in Lemma 2.6. Given any sequence $\sigma_{0}, \ldots, \sigma_{n-1}$ of comparable elements of $T$, let $\sigma_{n}$ be the least extension of $\sigma_{n-1}$ in $T$ and define $\hat{f}\left(\sigma_{0}, \ldots, \sigma_{n-1}\right)=f\left(\sigma_{0}, \ldots, \sigma_{n-1}, \sigma_{n}\right)$. Note that $\hat{f}$ is computable from $T$. By the induction hypothesis, there is a monochromatic tree $\hat{T}$ for $\hat{f}$ which is $\Pi_{n}^{0}$ in $T$. Since $\hat{T}$ is monochromatic for $f$, it remains only to show that $\hat{T}$ is $\Pi_{n+1}^{0}$. Since $\hat{T}$ is $\Pi_{n}^{0}$ in $T$, there is a $T$-computable $(n+1)$-place predicate $R$ such that for 
all $\tau$

$$
\tau \in \hat{T} \leftrightarrow \forall x_{1} \ldots Q x_{n} R\left(\tau, x_{1}, \ldots, x_{n}\right)
$$

where $Q x_{n} R$ is one of $\exists x_{n}$ and $\forall x_{n}$. The predicate $Q x_{n} R$ is computable in $T^{\prime}$ and hence in $0^{\prime \prime}$. Applying Post's hierarchy theorem (e.g., Theorem VIII(b) in $\S 14.5$ of [9]), we may replace $Q x_{n} R$ by either a $\Sigma_{3}^{0}$ or a $\Pi_{3}^{0}$ predicate, depending on whether $Q x_{n} R$ is $\exists x_{n}$ or $\forall x_{n}$. The resulting predicate is the required $\Pi_{n+1}^{0}$ definition of $\hat{T}$. $\dashv$

§3. Extensions and questions. We first note that all the preceding results can be extended to much broader classes of trees. For example, one could replace all the binary trees in this paper with ternary trees. The extensions are based on the fact that any subtree of $\mathbb{N}^{<\mathbb{N}}$ can be computably embedded into a copy of $2^{<\mathbb{N}}$. Consequently, $\mathrm{TT}_{k}^{n}$ can be extended as follows.

$\mathrm{ET}_{k}^{n}$ : If $T$ contains a subtree isomorphic to $2^{<\mathbb{N}}$ and $R$ is any subtree of $\mathbb{N}<\mathbb{N}$ containing an infinite path, then every $k$-coloring of $[T]^{n}$ contains a subtree $S$ isomorphic to $R$ such that $[S]^{n}$ is monochromatic.

All the reverse mathematics and computability results presented previously for $\mathrm{TT}_{k}^{n}$ hold identically for $\mathrm{ET}_{k}^{n}$. The requirement that $R$ contains an infinite path insures that the reversals and computability theoretic lower bounds hold.

We conclude with a short list of questions.

1. Is $\mathrm{TT}^{1}$ stronger than $\mathrm{B} \Pi_{1}^{0}$ ? Is it weaker than induction for $\Sigma_{2}^{0}$ formulas?

2. Does $\mathrm{TT}^{2}$ imply $\mathrm{ACA}_{0}$ ? Can Seetapun's result (as presented in [4]) be adapted to show that this is not the case?

3. What is the relative strength of $\mathrm{TT}^{2}$ and $\mathrm{TT}_{2}^{2}$ ? Can the work in [1] be adapted to address this?

4. To what degree can trees be replaced with other partial orders? Is there a Ramsey theorem on some class of partial orders where the theorem for pairs is equivalent to $\mathrm{ACA}_{0}$ ?

\section{REFERENCES}

[1] Peter A. Cholak, Carl G. Jockusch, and Theodore A. Slaman, On the strength of Ramsey's theorem for pairs, this JouRnAL, vol. 66 (2001), no. 1, pp. 1-55.

[2] W. Deuber, R. L. Graham, H. J. Prömel, and B. Voigt, A canonical partition theorem for equivalence relations on $\boldsymbol{Z}^{t}$, Journal of Combinatorial Theory, Series A, vol. 34 (1983), no. 3, pp. 331339.

[3] JefFry L. HiRSt, Combinatorics in subsystems of second order arithmetic, Ph.D. thesis, The Pennsylvania State University, 1987.

[4] Tamara Lakins Hummel, Effective versions of Ramsey's theorem: Avoiding the cone above $\mathbf{O}^{\prime}$, this JouRnaL, vol. 59 (1994), no. 4, pp. 1301-1325.

[5] CARL G. JockuSCh JR., Ramsey's theorem and recursion theory, this Journal, vol. 37 (1972), pp. $268-280$.

[6] Carl G. Jockusch Jr. and Robert I. Soare, $\pi_{1}^{0}$ classes and degrees of theories, Transactions of the American Mathematical Society, vol. 173 (1972), pp. 33-56.

[7] Тімотну H. McNicnoll, The inclusion problem for generalized frequency classes, $\mathrm{Ph} . \mathrm{D}$. thesis, The George Washington University, 1995.

[8] Keith R. Milliken, A Ramsey theorem for trees, Journal of Combinatorial Theory. Series A, vol. 26 (1979), no. 3, pp. 215-237. 
[9] HartLey Rogers JR., Theory of Recursive Functions and Effective Computability, McGraw-Hill Book Co., New York, 1967.

[10] Stephen G. Simpson, Subsystems of Second Order Arithmetic, Perspectives in Mathematical Logic, Springer-Verlag, Berlin, 1999.

THE GEORGE WASHINGTON UNIVERSITY

DEPARTMENT OF MATHEMATICS

2115 G STREET NW, WASHINGTON, DC 20052, USA

E-mail: jchubb@gwu.edu

URL: http://home.gwu.edu/ jchubb

APPALACHIAN STATE UNIVERSITY

DEPARTMENT OF MATHEMATICAL SCIENCES

BOONE, NC 28608, USA

E-mail: jlh@math.appstate.edu

$U R L$ : www.mathsci.appstate.edu/ jlh

LAMAR UNIVERSITY

DEPARTMENT OF MATHEMATICS

200 LUCAS BUILDING

BEAUMONT, TX 77710, USA

E-mail: mcnichollth@my.lamar.edu

$U R L$ : www.math.lamar.edu/faculty/monicholl/mcnicholl.asp 